\title{
Evaluation of the Limit State and Cyclic Life of Structural Materials under Thermal Fatigue
}

\section{A. P. Gopkalo}

Pisarenko Institute of Problems of Strength, National Academy of Sciences of Ukraine, Kiev, Ukraine

The relationship between the cyclic life and reduced stress in half-cycles of heating (compression) has been established, as well as the parameters defining the transition from the quasi-static to fatigue fracture mode and their threshold values. An approach has been proposed for the evaluation of cyclic life under thermal fatigue, which involves a preliminary prediction of the fracture mode and the use of calculation relationships corresponding to the predicted mode of fracture.

Keywords: reduced stress, quasi-static and fatigue fracture, cyclic life.

Introduction. A large number of present-day structures in operation are subject to the action of variable temperatures and mechanical loads. The experience of their operation shows that the limit state of the whole structure or its individual elements occurs due to fatigue crack propagation or inadmissible changes in the initial geometry (a change in the shape). Formation of a certain type of the limit state occurs by the accumulation of corresponding types of damages. Fatigue crack initiation and propagation are caused by cyclic alternating plastic deformation while the accumulation of quasi-static damages is associated with the processes of oriented plastic deformation (cyclic creep).

Peculiarities of the Material Damage under Isothermal and NonIsothermal Conditions. The life of materials under thermal fatigue is generally determined from the calculation relationships that correspond to a fatigue or quasi-static fracture mode under low-cycle mechanical loading under isothermal conditions. Attempts to use the same calculation relationships for thermal fatigue conditions yield an incorrect result despite the similarity of many processes under low-cycle mechanical fatigue in isothermal conditions and under thermal fatigue, such as the development of the local plastic strains and formation of cracks in the most heavily stressed zones (with the accumulation of the number of cycles); an increase in the stress and strain amplitudes leads to a decrease in the number of cycles to fracture; a similar effect of the stress and strain concentration, stress state mode, and many other factors is observed.

At the same time, there is a fundamental difference between the low-cycle mechanical fatigue and thermal fatigue. Under conditions of low-cycle mechanical loading at constant temperature, the same zones of the material are in turn subject to plastic tensile and compressive strains. In this case, the alternating cyclic elastic-plastic deformation is caused by a change in the resistance to the elastic and inelastic strains (the Bauschinger effect). Thermal stresses occur due to the thermal strain constraint, and therefore, they generally decrease rapidly with increasing temperature due to an increase in the plastic strain that has a tendency 
to be concentrated in the most heated zones of the body. In addition, the most heated zones of the body "absorb" the elastic-plastic strains that were to have occurred in the coolest zones due to the temperature dependence of the material mechanical characteristics.

Under thermal fatigue, the effects of the material hardening may not show depending on the level of the maximum temperature. Moreover, in the presence of the temperature gradient, tensile strain concentration can occur in some zones of the material, while in other ones the concentration of compressive strains takes place. Under thermal fatigue, due to repeated thermal loading, hot and cold working of the material is possible that has a great impact on the material strength and its subsequent properties. The temperature gradient in a temperature cycle that defines the elastic-plastic strain range is also of great importance here. At the maximum temperature in a cycle, the plastic strain value depends essentially on time and, therefore, the plastic strain is frequently accompanied by a considerable creep and stress relaxation, whereas at the minimum cycle temperature this dependence is weaker or absent. If the maximum cycle temperature is high enough, intensive development of intergranular deformation and fracture can occur, which are typical of the processes of creep and long-term fracture, with the development of diffusion, oxidation and other processes. At the values of the cycle minimum temperature below $150^{\circ} \mathrm{C}$, transgranular deformation and fracture primarily occur.

It is reasonable that the differences in the processes of the material deformation under isothermal and non-isothermal conditions considered here affect the material ability to resist cyclic deformation. Thus, if we consider the limiting amplitude diagrams for structural heat-resistant steels subjected to isothermal and non-isothermal loading conditions, the difference in the grouping of the points, which are representative of the corresponding mode of fracture, can be noted (Fig. 1). Under non-isothermal conditions, with the cycle stress ratio $R_{\sigma}$ varying from 0 to -0.32 , fatigue fracture takes place, whereas with $R_{\sigma}$ decreasing from -0.32 to -1 quasi-static fracture occurs. In contrast to non-isothermal conditions, under low-cycle loading and isothermal conditions, the fracture mode is independent of the cycle stress ratio and is defined by the values of the cycle stress amplitude and mean stress. The noted difference in the grouping of the points is due to the peculiarities of the deformation and fracture of materials under non-isothermal conditions indicated above and in [1].

Under conditions of thermal fatigue, both the values of the stresses generated in the material and the material mechanical characteristics vary during each thermal cycle. As an example, Fig. 2 illustrates the temperature dependences of the offset yield stress for some die steels, and Fig. 3 illustrates those of the thermal stresses occurring in these steels. Considering the reduced stresses in the materials (the ratio of the stress acting in the material to its offset yield stress) during one thermal cycle (Fig. 4), it should be noted that in the tensile half-cycles, the differences in the reduced stress values in materials are not as important as in the compressive half-cycles. The differences in the reduced stresses in materials during tensile and compressive half-cycles are, to a greater extent, due to the difference in the mechanical properties (Fig. 2) and, to a lesser extent, to the difference in the absolute values of the thermal stresses (Fig. 3). 

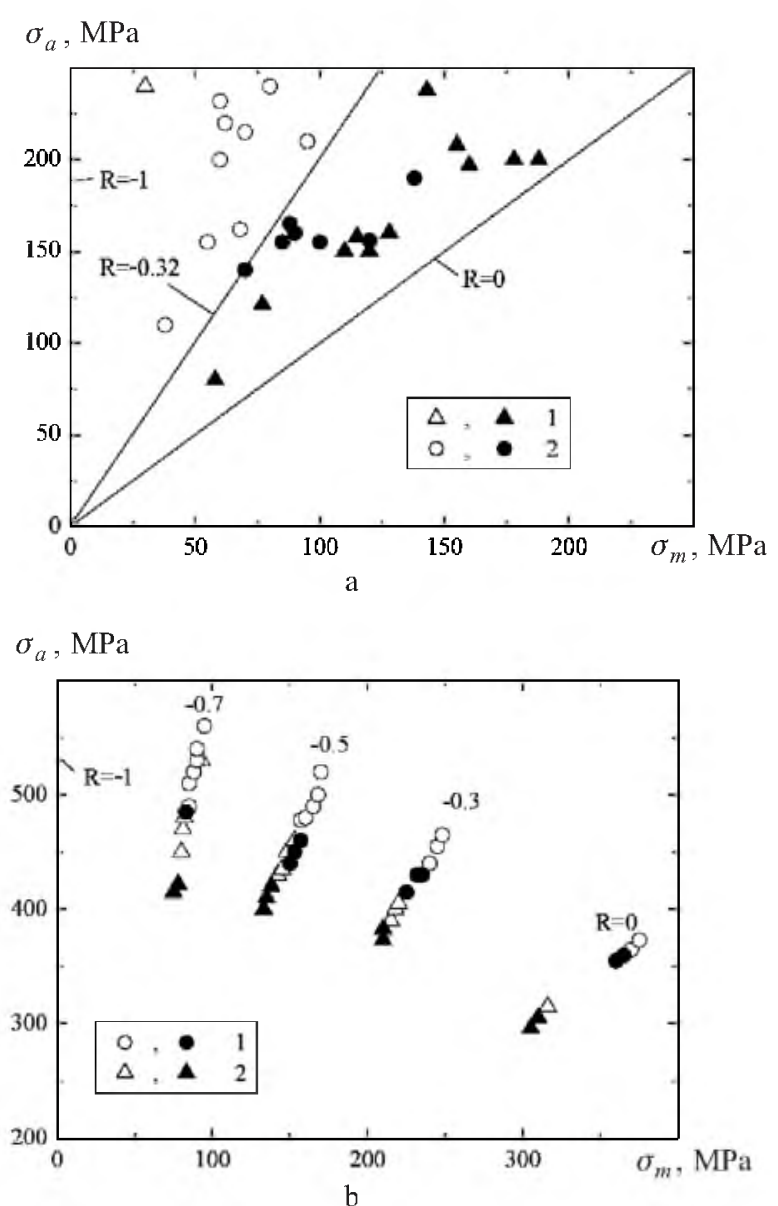

Fig. 1. The limiting amplitude diagrams for loading under non-isothermal [a: (I) steel $15 \mathrm{KhML}$, (2) steel $20 \mathrm{KhML}$ ] and isothermal [b: steel $15 \mathrm{Kh} 2 \mathrm{NMFA}$, (l) $\mathrm{T}=20^{\circ} \mathrm{C}$, (2) $\mathrm{T}=400^{\circ} \mathrm{C}$ ] conditions (open symbols stand for quasi-static fracture, solid symbols for the fatigue fracture).

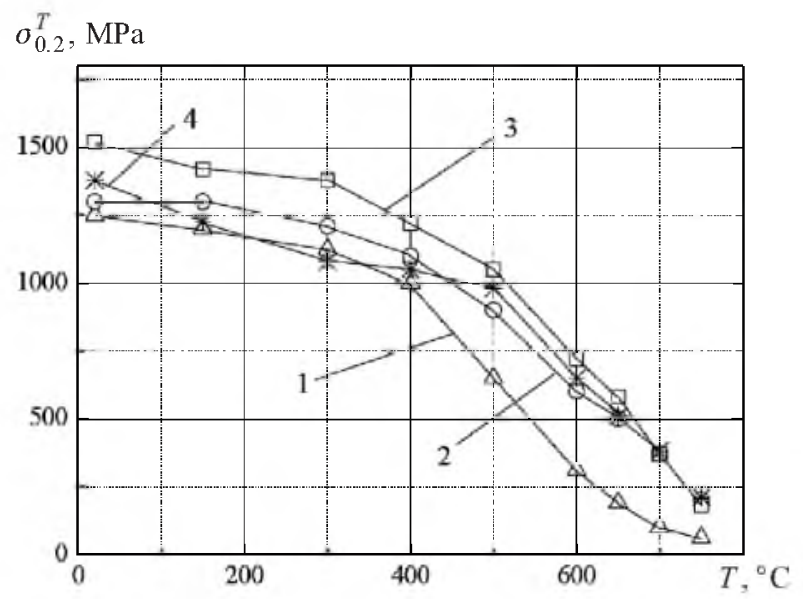

Fig. 2. Temperature dependence of the offset yield stress for the steels under study: ( $($ ) $5 \mathrm{KhNM}$; (2) 5KhMNAF; (3) Kh4M3AF; (4) 4Kh5MFAS. (The notations in Figs. 3-6 are the same as in Fig. 2.) 


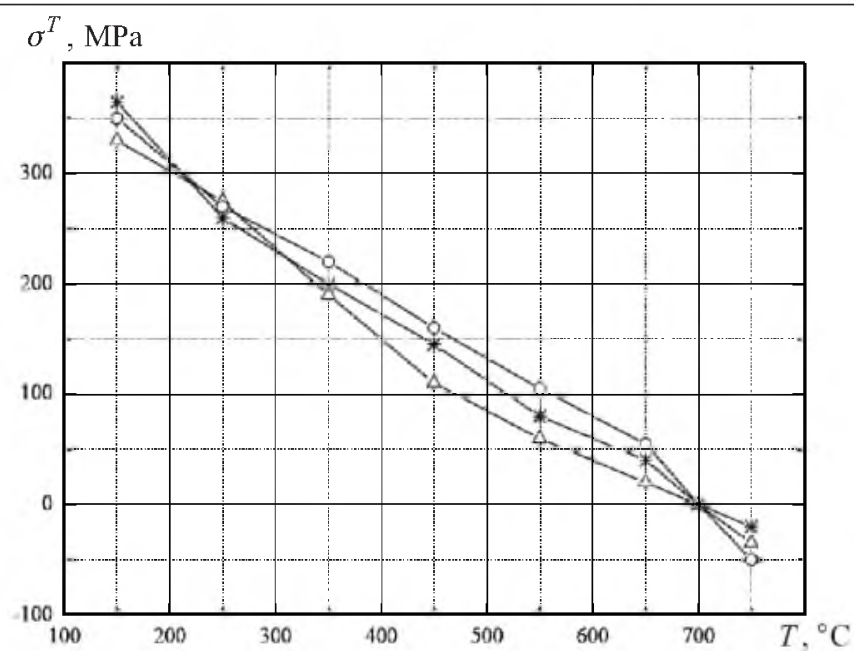

Fig. 3. Temperature dependence of the thermal stresses in a specimen within one thermal cycle at $2 \sigma=370 \mathrm{MPa}$

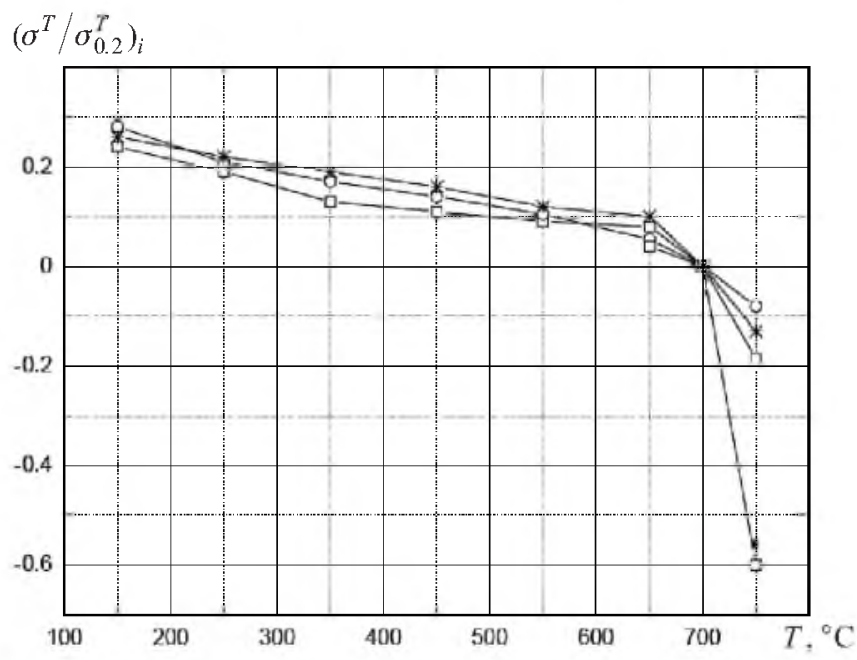

Fig. 4. Temperature dependence of the reduced stress in the steels under study within one thermal cycle at $2 \sigma=370 \mathrm{MPa}$.

Generalization of the results of experimental investigations into the resistance of a number of heat-resistant structural steels to deformation and fracture under thermal fatigue has shown that for $\sigma_{\min }^{T} / \sigma_{0.2}^{T}>0.8$ (the ratio of the compressive stress occurring at the maximum temperature of the thermal cycle to the offset yield stress determined at the same temperature), the mechanism of quasi-static damage is prevailing, whereas for $\sigma_{\min }^{T} / \sigma_{0.2}^{T}<0.6$, it is the mechanism of the fatigue damage [2]. At the steady-state creep rate $V_{c r}=1 \cdot 10^{-5} \mathrm{~mm} / \mathrm{mm} /$ cycle, a change in the mechanisms of damage accumulation occurs. At $V_{c r} \geq 1 \cdot 10^{-5}$ $\mathrm{mm} / \mathrm{mm} /$ cycle, quasi-static fracture takes place and at $V_{c r} \leq 1 \cdot 10^{-5} \mathrm{~mm} / \mathrm{mm} /$ cycle, fatigue fracture occurs. In this case, the threshold values of the steady-state creep rate, at which the change in the material damage mechanisms is observed, were 
confirmed by the test results for more than thirty various materials under isothermal low-cycle mechanical loading [3].

Traditional presentation of the fatigue curves in terms of stresses (Fig. 5a) or strains (Fig. 5b) shows that for every material there is its own thermal fatigue curve.
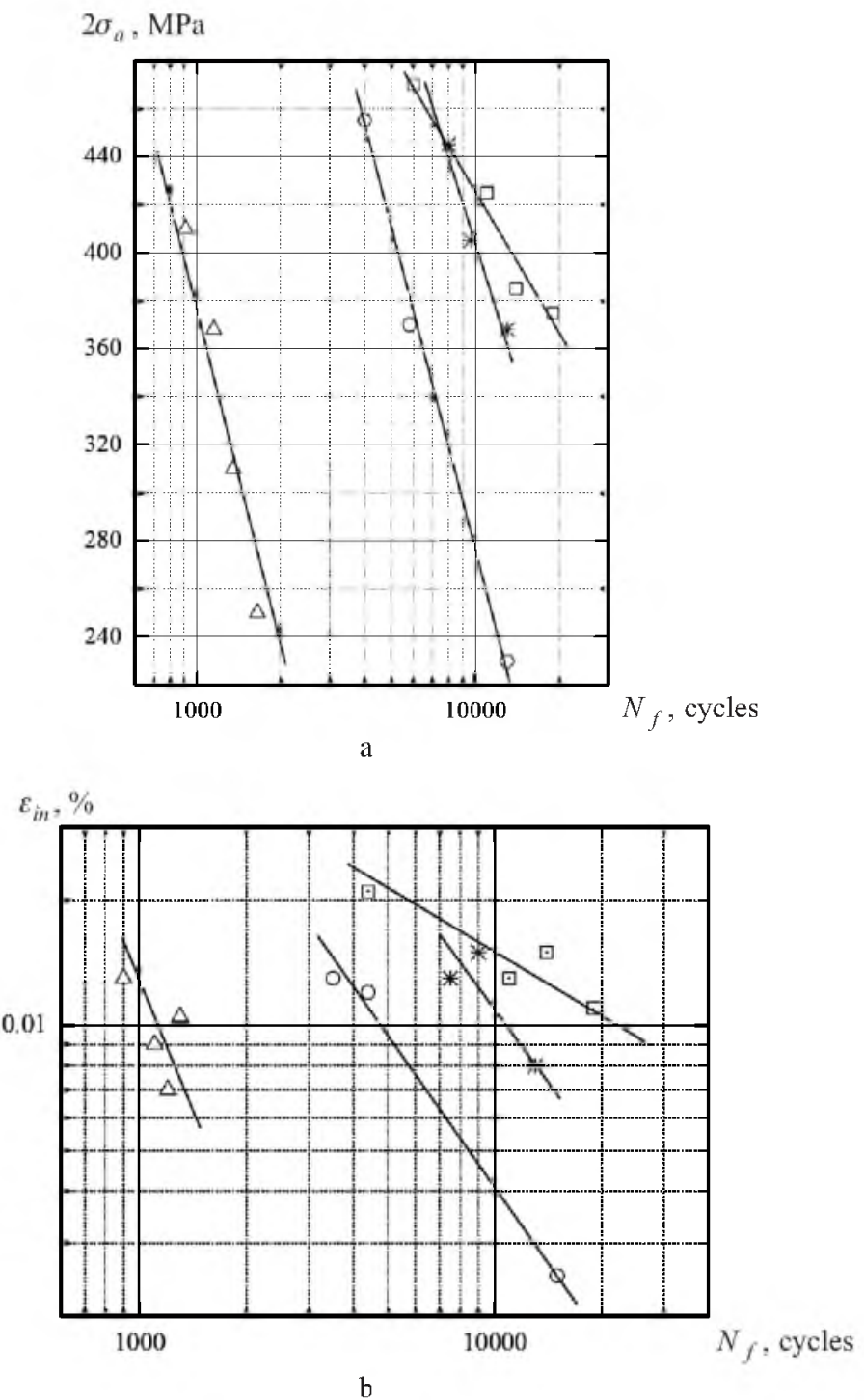

Fig. 5. Cyclic lifetime of the steels under study as a function of the stress range (a) and plastic strain range (b) for specimens heated from 150 to $750^{\circ} \mathrm{C}$.

If the same results are presented in the normalized coordinates (Fig. 6a, b), where $\sigma_{\max }^{T}$ is the maximum tensile stress occurring in the material under the action of the minimum temperature in a thermal cycle, $\sigma_{\min }^{T}$ is the minimum (compressive) stress occurring in the material under the action of the maximum temperature in a thermal cycle, $\sigma_{0.2}^{T}$ is the offset yield stress of the material at the 
minimum and maximum temperature of the thermal cycle, respectively, and $N_{f}$ is the number of cycles to fracture, the thermal fatigue curves differ essentially. But in the case where the same test results are presented in terms of $\left(\sigma_{\min }^{T} / \sigma_{0.2}^{T}\right)-N_{f}$, the experimental points for the steels under investigation are described by a single thermal fatigue curve, whereas when the results are presented in terms of $\left(\sigma_{\max }^{T} / \sigma_{0.2}^{T}\right)-N_{f}$, every material has its own thermal fatigue curve.

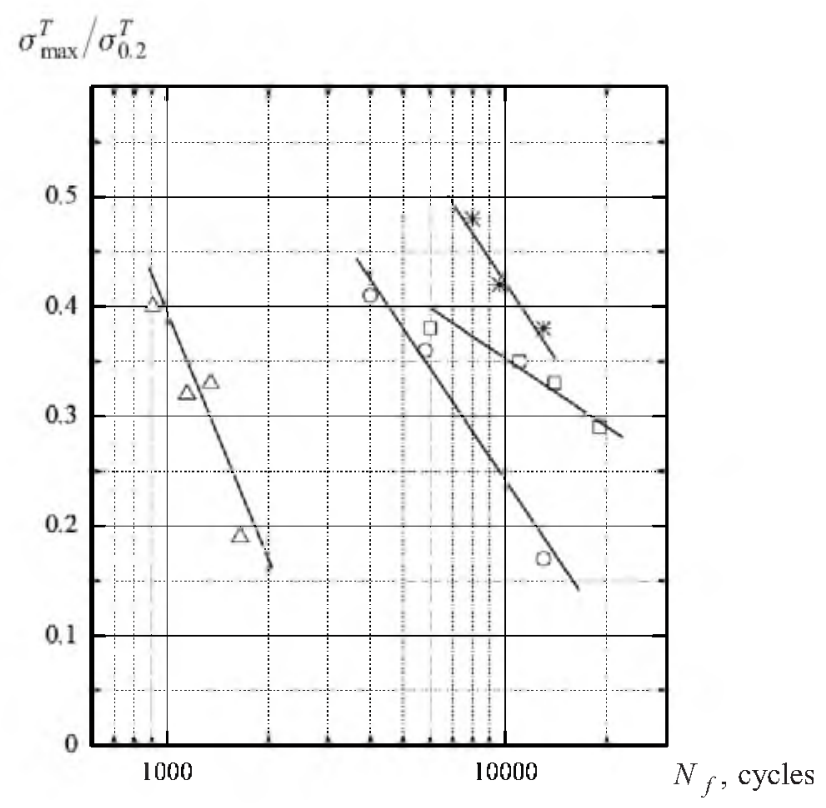

a

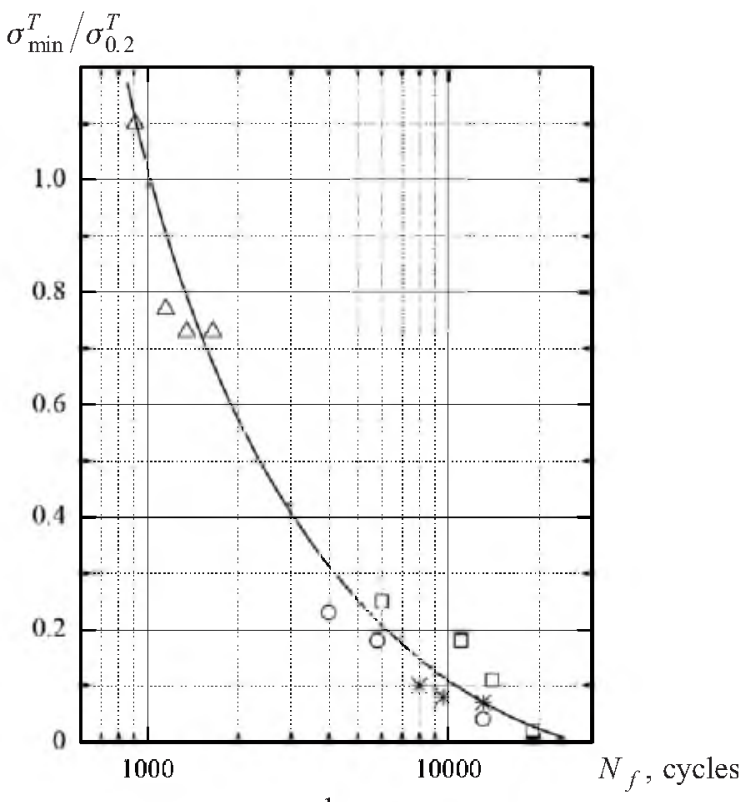

b

Fig. 6. Cyclic lifetime of the steels under study as a function of the reduced stress in tensile half-cycles (a) and compressive half-cycles (b) for specimens heated from 150 to $750^{\circ} \mathrm{C}$. 
This circumstance has made it possible to conclude that under thermal fatigue conditions, the most serious damage to the material occurs in the half-cycles of heating (compression) under the action of the maximum temperature in a thermal cycle, and the lifetime is governed, to a great extent, by the reduced stress in the material in these half-cycles.

It should be noted that under the action of the maximum temperature in the heating half-cycles, the material has lower mechanical characteristics than those at lower temperature in the cooling half-cycles. In the half-cycles of cooling, the maximum (tensile) stresses occur in the material; yet, their contribution to the total damage is not as significant as that of the half-cycles of heating (see Fig. 4). It is this circumstance that determines the availability of a single thermal fatigue curve for the materials under investigation in terms of the reduced stress versus number of cycles to fracture. This indicates that the reduced stress of the material in the half-cycles of heating (compression) is a more generalizing parameter that allows for the specific character of the loading conditions and the material properties.

Methods of Estimating Cyclic Life of Materials under Isothermal Conditions. As applied to the conditions of low-cycle mechanical fatigue, of interest are the Coffin-Manson method [4], Manson-Hirschberg method of universal slopes [5], the $10 \%$ rule [6], the Taira method [7], the method of strain amplitude separation, various cumulative damage rules $[9,10]$, as well as a number of stress-, strain-, energy-based and other models of the material damage. However, the practical use of the proposed methods for thermal fatigue conditions is limited by the complexity of obtaining the required data and does not always yield satisfactory results.

To estimate the cyclic life of materials under isothermal low-cycle loading, the following calculation relationships are widely used in the current engineering practice:

in the case of quasi-static fracture [11]

$$
N_{f}=\xi \frac{\varepsilon_{f}}{V_{c r}}
$$

and in the case of fatigue fracture [12]

$$
\varepsilon_{\text {in }} N_{f}^{k}=C \text {, }
$$

where $N_{f}$ is the number of the load cycles to fracture, $\xi$ is the reduced steady-state creep rate, which determines the degree of the material plasticity realization at the steady-state stage and characterizes the material capability to accumulate plastic strains at this stage, $V_{c r}$ is the steady-state creep rate, $\varepsilon_{f}$ is the available plasticity of the material, $\varepsilon_{\text {in }}$ is the plastic strain per cycle, and $k$ and $C$ are material constants.

The reduced steady-state creep rate $\xi$, which is determined from the generalized creep curves in the coordinates $\left(\varepsilon_{i} / \varepsilon_{f}\right)-\left(N_{i} / N_{f}\right)$, is a constant in the case of quasi-static fracture and is not such in the case of fatigue fracture [11]. 
Figure 7 presents the experimental data, which illustrate the relation between the parameter $\xi$ and the reduced stress values $\sigma_{\min }^{T} / \sigma_{0.2}^{T}$. From this figure it follows that in the region of quasi-static fracture $\left(\sigma_{\min }^{T} / \sigma_{0.2}^{T}>0.8\right)$ the values of the parameter $\xi$ are constant, whereas in the region of fatigue fracture $\left(\sigma_{\min }^{T} / \sigma_{0.2}^{T}<0.6\right)$ they are not.

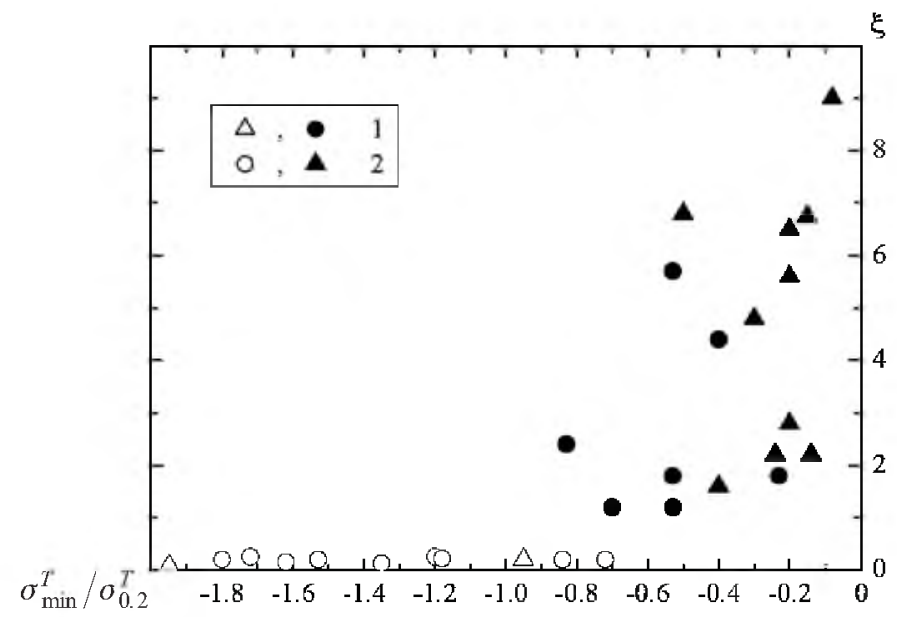

Fig. 7. Reduced steady-state creep rate versus the level of the reduced stress [(I) steel $15 \mathrm{KhML}$; (2) steel $20 \mathrm{KhML}$; open symbols - quasi-static fracture, solid symbols - fatigue fracture].

In view of the dual character of the materials fracture under conditions of thermal fatigue, when estimating cyclic life it is appropriate to perform preliminary prediction of the fracture mode (limit state) with the use of the threshold values of the parameters $\sigma_{\min }^{T} / \sigma_{0.2}^{T}$ or $V_{c r}$ established earlier [2] with subsequent selection of a calculation relationship corresponding to the real prevailing mechanism of damage accumulation.

The procedure for predicting the limit state and cyclic life of a material consists in the following. Based on the analysis of the service factors and physical-mechanical properties of the structure material, the temperature fields are determined in the most heavily loaded areas of the structure, and the thermal stress values are calculated with the use of a computational method. Then, using the obtained stress values, a possible limit state of the structure material (mode of fracture) is predicted.

Prediction of a possible limit state can also be made using the values of the steady-state creep rate. If the steady-state creep rate exceeds the threshold value, one should expect a quasi-static fracture (a change in the shape). If the values of the steady-state creep rate are below the threshold ones, one should expect a fatigue fracture (due to fatigue crack propagation).

\section{Conclusions}

1. Peculiar features of the reduced stress of materials in the half-cycles of compression and tension under thermal fatigue, which should be taken into account in engineering practice, have been established. 
2. The dependence of the cyclic life on the reduced stress in the half-cycles of heating (compression) has been determined, which, for structures operating under thermal fatigue, makes it possible to choose the material that would be most appropriate for the specified service conditions with a better justification and to conduct works aimed at creation of materials with specified properties of the resistance to thermal fatigue.

3. For operation under conditions of thermal loading, it is advisable to choose the materials whose offset yield stress does not decrease abruptly with a slight increase in temperature. The temperature range, within which an abrupt change in the material offset yield stress is possible, should be avoided.

4. Under conditions of thermal fatigue as well as under isothermal mechanical low-cycle loading, the parameter $\xi$, which enters into relationship (1), is a constant at quasi-static fracture, and is not a constant at fatigue fracture.

5. Comparison of the limiting amplitude diagrams obtained under isothermal and non-isothermal conditions shows that in the former case the material fracture mode is independent of the cycle stress ratio and is defined by the cyclic stress amplitude and the mean stress of the cycle, whereas in the latter case it depends on the cycle stress ratio.

6. To estimate the life of materials operating under conditions of cyclic temperature variation, it is possible to employ approaches involving a preliminary prediction of the fracture mode and the use of calculation relations corresponding to the predicted fracture mode.

7. Using the generalizing parameter $\sigma_{\min }^{T} / \sigma_{0.2}^{T}$, it is possible to specify the in-service conditions for operating structures by decreasing the reduced stress of the most critical elements and to extend the life of structures that have already exhausted their assigned service life.

1. A. P. Gopkalo, "An investigation of the features of loading in thermal fatigue," Strength Mater., 26, No. 10, 725-728 (1994).

2. V. T. Troshchenko, D. P. Sinyavskii, and A. P. Gopkalo, "Fracture criteria for metals under nonisothermal loading. Report No. 2," Ibid, 13, No. 12, 1450-1455 (1981).

3. A. P. Gopkalo and D. P. Sinyavskii, "On life prediction for structures operating under conditions of cyclic temperature variations," in: Reliability and Durability of Machines and Structures [in Russian], Issue 2, Kiev (1982), pp. 40-45.

4. L. F. Coffin, "On the thermal fatigue of steels," in: Heat-Resistant Alloys under Conditions of Varying Temperatures and Stresses [Russian translation], Gosenergoizdat, Moscow (1960), pp. 188-258.

5. S. S. Manson, "Fatigue: A complex subject - some simple approximations," Exp. Neck., 5, No. 7, 193-226 (1965).

6. S. S. Manson and G. R. Halford, "A method of estimating high-temperature low-cycle fatigue behavior of materials," in: Proc. Int. Conf. on Thermal and High-Strain Fatigue, Metals and Metallurgy Trust, London (1967), pp. 254270. 
7. S. Taira, "Lifetime of structures subjected to varying load and temperature," in: N. J. Hoff (Ed.), Creep in Structures, Academic Press, New York (1962), pp. $96-124$.

8. S. S. Manson, G. R. Halford, and M. H. Hirschberg, "Creep-fatigue analysis by strain-range partitioning," in: Proc. Symp. on Design for Elevated Temperature Environment, ASME (1971), pp. 12-28 (NASA TM X-67838, 1971).

9. R. A. Dul'nev, "Summation of static and cyclic effects on strength during thermocyclic loading," Strength Mater., 3, No. 10, 1234-1237 (1971).

10. V. T. Troshchenko, D. P. Sinyavskii, and A. P. Gopkalo, "Fracture criteria for metals under nonisothermal loading. Report No. 1," Ibid, 13, No. 12, 1445-1449 (1981).

11. V. A. Strizhalo, "Study on the regularities of the transition from quasistatic to fatigue rupture of light alloys under a few load cycles," Ibid, 6, No. 5, $567-573$ (1974)

12. V. A. Strizhalo, Cyclic Strength and Creep of Metals under Low-Cycle Loading at Low and High Temperatures [in Russian], Naukova Dumka, Kiev (1978).

Received 11. 06. 2008 